\title{
AVALIAÇÃO TARDIA DA MUCOSECTOMIA ESOFÁGICA COM CONSERVAÇÃO DA TÚNICA MUSCULAR EM PACIENTES COM MEGAESÔFAGO AVANÇADO
}

\author{
LONG TERM EVALUATION OF THE ESOPHAGEAL MUCOSECTOMY WITH \\ CONSERVATION OF MUSCULAR LAYER IN PATIENTS WITH ADVANCED \\ MEGAESOPHAGUS
}

\author{
José Luís Braga de Aquino, TCBC-SP1; Marcelo Manzano Said²; Pedro Ricardo Fernandes³
}

\begin{abstract}
RESUMO: Objetivo: Estudar retrospectivamente, por meio de uma avaliação tardia, a técnica de mucosectomia esofágica em pacientes com megaesôfago avançado. Método: Foram estudados 50 pacientes portadores de megaesôfago avançado submetidos à mucosectomia esofágica com conservação da túnica muscular no período de janeiro de 1991 a dezembro de 1997. Em todos os pacientes foi realizada avaliação tardia entre 6 e 15 anos de pós-operatório. A idade variou de 30 a 69 anos, com média de 53,5 anos, com predominância do sexo masculino em 32 pacientes (64\%). Em todos os doentes, o estudo se fez em relação à avaliação clínica no que concerne à qualidade da deglutição, à presença ou não de regurgitação, a alterações no hábito intestinal, à evolução ponderal, à satisfação com a cirurgia e ao retorno à atividade normal. Também foi realizada a avaliação morfológica e funcional pelo estudo radiológico contrastado, pela endoscopia digestiva alta e pela tomografia computadorizada de tórax. Na avaliação clínica, morfológica e funcional foi realizada uma avaliação global representada pela somatória dos valores numéricos decorrentes da análise de cada um dos parâmetros, sendo o resultado final considerado bom, ótimo e regular e mau de acordo com o total dos pontos obtidos. Resultados: Na avaliação clínica global, 44 pacientes (88\%) apresentaram classificação entre ótima e boa, e o restante regular para mau. Na avaliação radiológica global, ótima e boa estiveram presentes em 47 pacientes $(94 \%)$ e regular e mau no restante. Na avaliação endoscópica global, 45 pacientes (90\%) obtiveram resultados ótimos para bons, e o restante regular para mau. Em relação à tomografia computadorizada de tórax, a avaliação global foi realizada em 31 pacientes sendo considerada ótima e boa em todos eles. Conclusão: A avaliação tardia do pós-operatório evidenciou que a maioria dos pacientes apresentou avaliação boa e ótima, tanto na avaliação clínica como na morfológica e funcional (Rev. Col. Bras. Cir. 2007; 34(1): 09-15).
\end{abstract}

Descritores: Esôfago/ cirurgia; Esofagectomia; Acalasia esofágica

\section{INTRODUÇÃO}

A esofagectomia subtotal sem toracotomia, embora não seja a terapêutica cirúrgica ideal para o megaesôfago avançado, tem ainda tido grande aceitação ${ }^{1-2}$.

A vantagem dessa via de acesso é a de evitar o comprometimento da dinâmica pulmonar. Entretanto, pode haver a abertura da pleura e, como conseqüência, o desenvolvimento de hemo ou hidropneumotórax, acarretando maior morbidade pós-operatória ${ }^{3-5}$. A lesão pleural geralmente ocorre durante o descolamento mediastinal do esôfago que, com intensa periesofagite acarreta aderências da víscera aos folhetos pleurais.

Além disso, é bem notório que, no megaesôfago avançado, a esofagite de estase predisponha a instalação de lesões pré-neoplásicas, podendo evoluir até carcino$\mathrm{ma}^{6-7}$.

Tendo em vista tais considerações é que se cogitou um método que possibilitasse a retirada da mucosa e submucosa do esôfago por meio da sua invaginação completa, através da via cervicoabdominal combinada sem toracotomia e conservando por inteiro a túnica mus- cular esofágica. Desse modo, seria feita a profilaxia de lesões mucosas pré-neoplásicas e evitando as complicações da dissecção e do descolamento do esôfago no mediastino.

Como etapa preliminar da sua aplicação na área clínica, realizou-se estudos no cão, demonstrando a sua viabilida$\mathrm{de}^{8}$. Em fase posterior, estudos em cadáver humano demonstraram a exequiibilidade do método em questão. Assim, amparados por essa verificação experimental, iniciou-se a nossa experiência clínica com boa evolução na avaliação inicial ${ }^{9} \mathrm{e}$ mais recentemente, com maior vivência clínica, demonstramos que esse procedimento técnico apresenta baixa morbidade, principalmente o hemopneumotórax, com incidência menor que $10 \%{ }^{10}$.

Entretanto, a grande dúvida era o que poderia ocorrer a longo prazo com esses pacientes submetidos a essa técnica, como fibrose da túnica muscular do esôfago ao nível do mediastino comprometendo a evolução dos pacientes.

O objetivo desse estudo é avaliar, no pós-operatório tardio, uma série de pacientes com megaesôfago avançado submetidos à mucosectomia esofágica.

1. Professor Titular de Clínica Cirúrgica da Faculdade de Medicina da PUC-Campinas.

2. Cirurgião Torácico do Hospital Celso Pierro - PUC-Campinas.

3. Ex-residente de Cirurgia Geral do Hospital Celso Pierro - PUC-Campinas.

Recebido em 14/07/06

Aceito para publicação em 15/08/06

Conflito de interesses: nenhum

Fonte de financiamento: nenhuma

Trabalho realizado no Departamento de Cirurgia da Faculdade de Medicina da PUC- Campinas. 


\section{MÉTODO}

Foram estudados 50 pacientes adultos portadores de megaesôfago Grau III/IV, submetidos à mucosectomia esofágica com conservação da túnica muscular no Serviço de Cirurgia do Hospital e Maternidade Celso Pierro - PUC-Campinas, no período de janeiro de 1991 a dezembro de 1997, segundo técnica que descrevemos e padronizamos ${ }^{9,10}$. Em todos os pacientes foi realizada a avaliação tardia entre fevereiro de 2004 a novembro de 2005.

Em todos os doentes foi realizada avaliação clínica entre 6 e 15 anos após a operação, incluindo os seguintes parâmetros:

- qualidade de deglutição;

- ocorrência de regurgitação gastroesofágica pósprandial e em decúbito;

- alteração do hábito intestinal;

- evolução ponderal;

- satisfação com a intervenção;

- retorno às atividades profissionais.

Para uma análise clínica mais criteriosa, foi criada uma escala numérica para cada parâmetro estudado.

A) Qualidade de deglutição

2: Normal;

1: Disfagia ocasional;

0: Disfagia freqüente.

B) Regurgitação

2: Ausente;

1: Ocasional;

0 : Freqüente.

C) Hábito intestinal

2: Inalterado;

1: Diarréia/constipação ocasional;

0 : Diarréia/constipação freqüente.

D) Evolução ponderal

2: Aumentada;

1: Inalterada;

0: Diminuída.

E) Satisfação com a intervenção cirúrgica

1: Sim;

0: Não.

F) Retorno à atividade profissional

1: Sim;

0: Não.

A avaliação clínica global foi representada pelo somatório dos valores numéricos decorrentes da análise de cada um dos parâmetros clínicos, sendo o resultado final considerado de acordo com o total de pontos obtidos, a saber:

10 e 9 Ótimo

8 e 7 Bom
6 e 5

Regular

$4,3,2,1$ e 0 Mau

Avaliação morfológica e funcional também foi realizada no mesmo intervalo de tempo, mediante exame radiológico contrastado convencional, endoscopia do sistema digestivo alto e tomografia computadorizada de tórax. O estudo radiológico contrastado do esôfago, do estômago interposto e do duodeno foi realizado em todos os doentes por técnica radiológica semelhante ao estudo convencional. Foram analisados os aspectos referentes à junção esofagogástrica, ao estômago interposto e à transição gastroduodenal. Foi observada a perviedade da anastomose esofagogástrica, avaliando-se a presença ou não de estenose que quando presente, foi correlacionada à qualidade de deglutição. A esse parâmetro foram atribuídos valores numéricos, a saber:

3

Ausência

2 Estenose discreta

1 Estenose moderada

0 Estenose intensa

Quanto ao estômago interposto, foi analisada a ocorrência ou não de compressão em relação à progressão da substância de contraste. Foi considerada como compressão a presença de estreitamento com estase a montante.

A ocorrência de compressão do estômago interposto foi correlacinada à qualidade de deglutição, sendo atribuídos estes valores numéricos:

1

0

Ausência de compressão Presença de compressão

Avaliou-se ainda a perviedade adequada ou não da transição gastroduodenal, pela presença de estase de contraste ao nível do estômago distal. Seu comprometimento foi correlacionado à presença ou não de regurgitação, optandose também por escala em números:

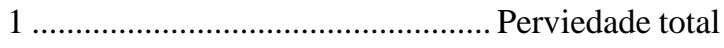

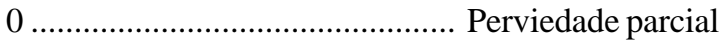

Como na apreciação clínica, para se ter uma avaliação radiológica mais global, foi considerado o somatório dos valores numéricos dos três parâmetros assinalados, a saber:

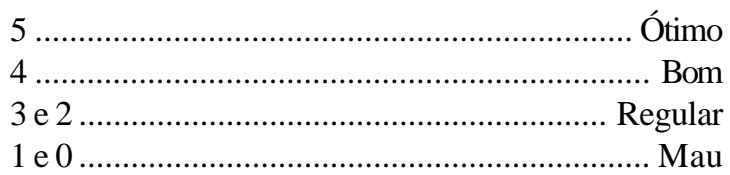

A avaliação endoscópica foi realizada em todos os pacientes com o videoendoscópio Fujinon, modelo E G-7-H R2 superimagem, de 10,2 mm de diâmetro, com preparo e exames conforme sistematização padronizada pelo Serviço de Endoscopia Digestiva do Hospital e Maternidade Celso Pierro. 
Foram analisados aspectos relacionados à perviedade da anastomose esofagogástrica, da transição gastroduodenal e as alterações macroscópicas presentes na mucosa do coto esofágico e do estômago interposto.

A presença ou não de estenose da anastomose esofagogástrica foi correlacionada à qualidade de deglutição, ao qual se atribuíram os seguintes valores:

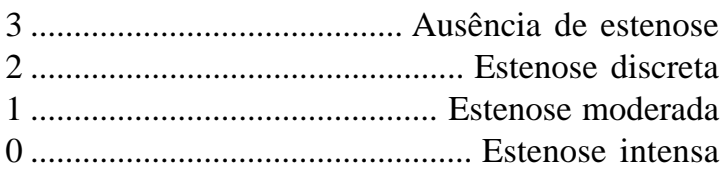

O calibre da transição gastroduodenal, avaliado pela ultrapassagem do aparelho de videoendoscopia pela região pilórica, foi correlacionado à presença de regurgitação. Também foram atribuídos valores numéricos, a saber:

$$
1
$$

Perviedade total

0 Perviedade parcial

Em relação à avaliação macroscópica da mucosa esofagogástrica, foram estimadas as alterações inflamatórias da mucosa do esôfago cervical remanescente, sendo-lhes atribuídos os valores abaixo:

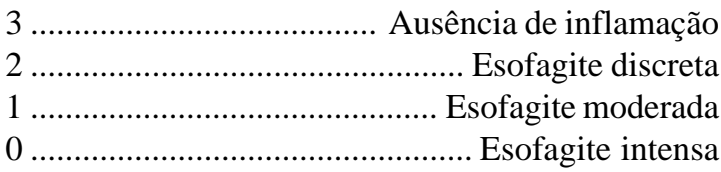

A mesma avaliação foi efetuada com a mucosa do estômago interposto:

3

2

1

0

Como nas apreciações anteriores, foi considerado o somatório dos valores numéricos dos três parâmetros, para se ter uma análise endoscópica mais criteriosa:

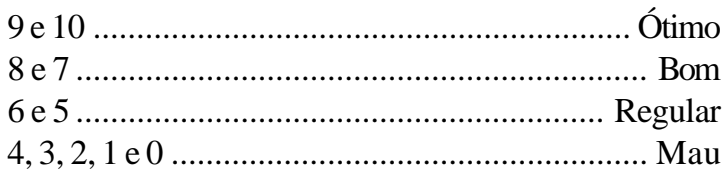

A avaliação tomográfica de tórax foi realizada com cortes axiais de $10 \mathrm{~mm}$ de espessura e espaçamento de 10 e 30 mm, após a ingestão de contraste de solução iodada diluída, segundo o Serviço de Radiologia e Métodos de Imagem do Hospital Celso Pierro - PUC-Campinas.

Nessa avaliação, foi estudado o estômago transposto por via transmediastinal por dentro da túnica muscular esofágica, sendo analisado aspectos referentes à presença de coleção intramediastinal ou entre o estômago e a parede muscular esofágica; presença ou ausência de compressão de al- gum órgão intramediastinal; características da túnica muscular esofágica em relação à sua individualização ou não; presença ou ausência de deslocamento do conjunto estômago/ túnica muscular, atribuindo-se-lhes os seguintes valores numéricos: lar esofágica:

Coleção intramediastinal/estômago e parede muscu-

1 . Ausência

0 Presença

Compressão de órgão intramediastinal:

1 Ausência

0 Presença

Individualização da túnica muscular esofágica:

1 Sim

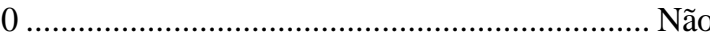

Deslocamento do conjunto estômago/túnica muscular esofágica:

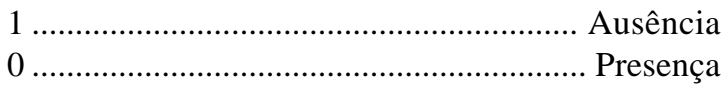

Para uma avaliação global mais criteriosa, foi estabelecido também um somatório dos valores obtidos em cada um dos parâmetros, a saber:

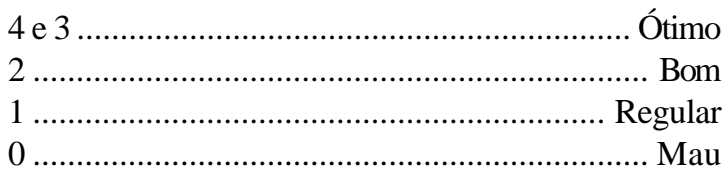

\section{RESULTADOS}

\section{Qualidade de deglutição}

A idade dos pacientes variou de 30 a 69 anos, com maior freqüência entre 50 e 59 anos. Quanto ao sexo, 32 eram do masculino (64\%) e 18 do feminino (36\%).

Trinta e oito pacientes $(76 \%)$ evoluíram com deglutição normal, oito (16\%) com disfagia ocasional para sólidos e quatro (8\%) com disfagia freqüente para sólidos.

\section{Regurgitação}

Trinta e dois pacientes (64\%) evoluíram sem regurgitação, 17 (34\%) com regurgitação ocasional e um (2\%) com regurgitação freqüente e quase sempre relacionada ao decúbito, principalmente no período pós-prandial.

\section{Hábito intestina}

Vinte e nove pacientes (58\%) não apresentaram nenhuma alteração no hábito intestinal; 17 (34\%) apresentaram diarréia ou constipação ocasional e quatro (8\%) constipação freqüente. Estes quatro últimos pacientes apresentaram na avaliação pré-operatória megacólon, sendo dois deles subme- 
tidos à sigmoidectomia, com reconstrução do trânsito por abaixamento retroretal do colo descendente após 35 a 48 meses da mucosectomia esofágica.

\section{Evolução ponderal}

$\mathrm{Na}$ avaliação clínica da evolução ponderal, 39 pacientes $(78 \%)$ apresentaram aumento de peso no pós-operatório, oito (16\%) mantiveram peso semelhante e três $(6 \%)$ diminuição.

\section{Satisfação com o tratamento cirúrgico}

Quarenta e seis pacientes $(92 \%)$ afirmaram estar satisfeitos, apresentando uma qualidade de vida muito melhor em relação ao pré-operatório. Para três pacientes $(6 \%)$, a intervenção operatória diminuiu pouco os sintomas em relação ao pré-operatório e para o paciente restante $(2 \%)$, além de continuar a apresentar os mesmos sintomas do préoperatório, a sua qualidade de vida estava inferior ao esperado.

\section{Retorno às atividades profissionais}

Foi inquerido aos pacientes se no pós-operatório retornaram às atividades profissionais que exerciam previamente ao ato cirúrgico. Quarenta e quatro pacientes $(89 \%)$ responderam positivamente, três $(6 \%)$ referiram que embora estivessem se sentindo satisfeitos, achavam que não tinham condições de retornar às atividades que exerciam, pois seus trabalhos exigiam esforços moderados a grandes, três $(6 \%)$ referiram que embora não exercessem atividades que exigissem esforços, a intervenção cirúrgica os tornaram limitados, devido a continuarem com os sintomas semelhantes ao préoperatório, não conseguindo exercer sua atividades profissionais habituais.

\section{Avaliação clínico global}

Vinte e cinco pacientes $(50 \%)$ apresentaram avaliação ótima, $19(30 \%)$ boa, quatro (8\%) regular e dois (4\%) má.

\section{Avaliação radiológica}

Em 43 pacientes (86\%) não houve nenhuma anormalidade ao nível da junção esofagogástrica, em quatro $(8 \%)$ se demonstrou estenose discreta e nos três $(6 \%)$ restantes, estenose moderada.

Quanto aos achados radiológicos de compressão do estômago interposto, em 48 pacientes (96\%) não houve anormalidade alguma, em dois (4\%) houve compressão gástrica junto à região cervical.

Em 41 pacientes $(82 \%)$ houve boa perviedade da transição gastroduodenal e em nove $(18 \%)$ perviabilidade parcial.

A avaliação radiológica global revelou que 42 pacientes $(84 \%)$ obtiveram apreciação ótima, cinco (10\%) boa, um (2\%) regular e dois $(4 \%)$ má.

A avaliação endoscópica, em 42 pacientes (84\%) não revelou nenhuma alteração ao nível da anastomose; em cinco (10\%) demonstrou estenose discreta e em três (6\%), estenose intensa.

Em 42 pacientes $(84 \%)$ houve perviedade total e em oito $(16 \%)$ parcial da transição gastroduodenal.
Em 29 pacientes (59\%) não houve inflamação ao nível da mucosa do esôfago cervical remanescente; 18 (36\%) apresentaram esofagite discreta a moderada e os três $(6 \%)$ restantes esofagite intensa, sendo que em um deles se confirmou a presença de mucosa de Barret, pelo estudo anatomopatológico.

Em 16 pacientes (32\%) não ocorreu nenhuma alteração da mucosa do estômago interposto, pela avaliação endoscópica; em 17 (34\%) houve gastrite discreta, em 14 (28\%) gastrite moderada e nos três $(6 \%)$ restantes, gastrite intensa.

A avaliação endoscópica global demonstrou que em 22 pacientes (44\%), a avaliação foi ótima, em 27 (46\%) boa, em três $(6 \%)$ regular e em dois $(4 \%)$ má.

tórax

Avaliação da tomografia axial computadorizada de

A tomografia computadorizada de tórax foi realizada em 31 pacientes. Vinte e oito pacientes $(90,3 \%)$ não apresentaram nenhuma coleção. Em três deles $(9,7 \%)$ havia coleção entre o estômago e a túnica muscular esofágica, sendo em dois localizados em posições supra carinais e de pequeno tamanho, e outra de maior diâmetro infracarinal. Nenhum dos três pacientes apresentou sintomas referentes a estas coleções. Paciente algum (0\%) apresentou indícios de compressão de qualquer órgão intramediastinal pelo conjunto estômago/túnica muscular.

Nos 31 pacientes em que se analisaram as características da túnica muscular do esôfago, esta se mostrou bem individualizada em 30 delas $(96,8 \%)$ e praticamente aderida ao estômago interposto, mas sem ocasionar compressão.

Em três $(9,7 \%)$ pacientes se verificou deslocamento do conjunto estômago/túnica muscular esofágica, mas sem nenhuma repercussão clínica.

Em $28(90,3 \%)$ pacientes a avaliação global da tomografia computadorizada de tórax foi ótima e em três $(9,71 \%)$ foi boa.

\section{DISCUSSÃO}

A mucosectomia esofágica com conservação da túnica muscular para o tratamento do megaesôfago avançado já demonstrou na avaliação pós-operatória precoce ser de grande valia por apresentar baixo índice de morbidade, principalmente no tocante às complicações pleuro-pulmonares ${ }^{9,10}$. Daí a importância de uma avaliação mais tardia, para tentar demonstrar se este procedimento técnico também é adequado, a longo prazo.

Assim, na avaliação clínica da qualidade de deglutição esta se mostrou normal na maioria dos enfermos. Nos quatro pacientes, cuja qualidade de deglutição se revelou inferior ao normal, havia, em três deles, estenose da anastomose esôfago-gástrica cervical, demonstrado pela avaliação radiológica e endoscópica. Um aspecto importante no que se refere à deglutição nesses três pacientes é o fato de que em dois deles havia compressão do estômago, como se observou na escala de avaliação radiológica. Isso deve ter acontecido pela fibrose que se instala no local, entre o estômago e as estruturas vizinhas, sendo que um paciente também 
já ter sido submetido previamente à cervicotomia exploradora por trauma.

Assim, um desses três pacientes com dilatação endoscópica periódica obteve melhora satisfatória da deglutição até com a avaliação realizada com 120 meses de pós-operatório os outros dois ainda continuaram a apresentar comprometimento evidente da deglutição com 132 e 148 meses de pós-operatório, quando da última avaliação.

Os outros cinco pacientes apresentaram estenose discreta evidenciada pelo estudo endoscópico e radiológico e pela disfagia ocasional. Este fato talvez seja explicado pela possível alteração funcional do coto esofágico remanescente. Essa alteração de motricidade do esôfago superior tem sido demonstrada por outros autores, até em indivíduos normais, sendo mais evidente nos pacientes chagásicos ${ }^{11,12}$.

Assim, o parâmetro da deglutição refletindo sua boa qualidade com a técnica cirúrgica empregada é também observado por outros quando da gastroplastia transmediastinal posterior, após a esofagectomia sem toracotomia para megaesôfago avançado ${ }^{13-15}$.

Um outro parâmetro clínico que se destacou na evolução tardia foi a regurgitação, que apresentou uma incidência similar às séries que relatam a gastroplastia após esofagectomia sem toracotomia por outras técnicas ${ }^{13,16,17}$. Dos 17 pacientes que a apresentavam no pós-operatório, em nove deles, houve correlação com os achados radiológicos contrastados e endoscópicos após demonstrarem comprometimento parcial da perviedade gastroduodenal. Em vista de apresentarem regurgitação de modo ocasional e mais relacionada ao decúbito, a terapêutica de orientação alimentar fez com que os pacientes revelassem boa evolução até a última avaliação entre 96 a 142 meses de pós-operatório.

Alguns autores já demonstraram que o refluxo gastroesofágico após a esofagogastroplastia ocorre quando a anastomose é realizada cranialmente ao esfíncter superior do esôfago ou quando o doente permanece em decúbito dorsal após as refeições ou ainda quando há obstáculos ao esvaziamento gástrico ${ }^{18}$.

Nos outros oito pacientes com regurgitação ocasional ao decúbito após as refeições não havia comprometimento orgânico ao nível do trajeto do estômago ou da transição gastroduodenal, avaliadas por meio de parâmetros radiológicos e endoscópicos, e deve ter ocorrido por comprometimento funcional gástrico conseqüente à vagotomia, apesar da piloromiectomia realizada.

O efeito da secção vagal sobre a motilidade gástrica tem sido bem estudada, resultando na maioria das vezes, em hipotonia com redução do número de potência das contrações do corpo e antro gástrico ${ }^{13,19}$. Chaib et $a l .^{13}$, no estudo de uma série de pacientes submetidos à esofagectomia sem toracotomia, a maioria por megaesôfago avançado com reconstrução do trânsito com o estômago transmediastinal posterior, demonstrou, por meio de estudos cineradiográficos, que, em decúbito dorsal, o contraste permanecia parado no estômago por tempo bastante prolongado. Com o paciente em posição ortostática, havia imediata descida do contraste sob a ação da gravidade, esvaziando o estômago completamente com pequena participação de sua musculatura.
Apesar da regurgitação se mostrar pouco intensa e ocasional, havia na avaliação endoscópica, $68 \%$ e $42 \%$ dos doentes com estase que tinham, respectivamente, inflamação do estômago interposto e do esôfago cervical remanescente, sendo, na maioria, discreto ou moderado, embora um paciente tinha apresentado esôfago de Barret, com 113 meses de pósoperatório. Este fato enfatiza a importância do acompanhamento a longo prazo desses pacientes, devido à possibilidade, inclusive de malignização. Essa alteração ao nível do epitélio gástrico e esofágico também tem sido demonstrada por outros autores quando da esofagectomia sem toracotomia com gastroplastia transmediastinal para o tratamento do megaesôfago avançado ${ }^{17,20,21}$.

A baixa repercussão dos pacientes com regurgitação e com sintomas pouco intensos conseqüentes à gastrite $\mathrm{e}$ esofagite, indica que com o tempo, o estômago se adapta funcionalmente à nova situação anatômica, mostrando-se em geral com o aspecto tubular.

No que se refere à avaliação tardia do hábito intestinal, demonstrou-se que embora a incidência de diarréia não fosse desprezível, a maioria foi ocasional, revelando boa evolução com o tratamento clínico quando da avaliação realizada aos 96 e 109 meses do pós-operatório. Essa alteração do hábito intestinal também é relatada por outros autores, quando realizam a esofagectomia nos pacientes com megaesofago independentemente da técnica que se emprega, e geralmente vale conseqüente à secção vagal e piloroplastia, pois esta também pode contribuir para o esvaziamento gástrico rápido 13, 16, 17,20,21.

Apesar da evolução ponderal não se relacionar diretamente à técnica de ressecção, esta, todavia, sendo bem padronizada, com baixa morbidade e favorecendo uma reconstrução do trânsito bem adequada, predispõe ao aumento progressivo do peso corporal no pós-operatório tardio. Isso ficou bem evidente na nossa série, pois $78 \%$ dos pacientes apresentaram aumento do peso pós-operatório em relação ao pré-operatório. Dos oito pacientes cujo peso pós-operatório foi semelhante ao pré-operatório, seis deles tinham o peso dentro da faixa de normalidade.

Nos três pacientes em que a evolução ponderal foi abaixo do esperado, os mesmos apresentavam disfagia conseqüente ao comprometimento orgânico da anastomose esofagogástrica além de que em dois deles também houve compressão do estômago interposto.

Esses dados são semelhantes às outras séries de esofagectomia sem toracotomia por megaesôfago, em que a maioria dos doentes apresenta boa evolução ponderal com aumento do peso no pós-operatório tardio ${ }^{13-16,20,22}$.

A baixa repercussão apresentada pela alteração clínica em alguns pacientes demonstra que a mucosectomia esofágica pelo deslocamento submucoso com gastroplastia transmediastinal posterior por dentro da túnica muscular, ofereceu boa qualidade de vida em longo prazo. E isso se tornou mais evidente já que $92 \%$ dos pacientes se disseram satisfeitos com a cirurgia e $88 \%$ retornaram às atividades que exerciam antes do ato cirúrgico proposto.

Uma consideração que deve ser levada em conta é em relação aos achados da tomografia computadorizada de tórax. Embora esse procedimento não tenha sido realizado em 
todos os pacientes, naqueles em que o foi, demonstrou que três pacientes com o estômago transposto por dentro da túnica muscular remanescente, apresentavam coleção de líquidos, que poderia ter ocorrido como conseqüência da aderência de fragmentos de mucosa à túnica muscular. Contudo, tanto na avaliação intra-operatória como no exame histopatológico, ficou evidenciada a retirada por inteiro da mucosa ${ }^{10}$. Assim, a irritação constante da serosa do estômago sobre a túnica muscular do esôfago, associada às contrações peristálticas da parede gástrica, talvez tenha levado à formação de espaços que evoluiram para a criação de coleção líquida.

Pelo fato dos três pacientes não mostrarem sintoma algum consequiente a essa alteração, o tratamento foi apenas conservador, com boa evolução até a última avaliação pós-operatória aos 142, 148 e 172 meses. Outro fato que ficou bem evidente na tomografia computadorizada do tórax, nesse subgrupo de pacientes, é que nenhum deles apresentou compressão gástrica pela túnica muscular, fato que já ficou demonstrado pelas observações endoscópicas e radiológicas.

Assim, a tomografia computadorizada de tórax realizada em $62 \%$ dos pacientes do presente estudo inferiu que mediante esse parâmetro, mais uma vez se demonstrou que a técnica de ressecção da mucosa pelo plano submucoso é possível de realização sem complicações tardias.

Assim, pelos resultados obtidos pela avaliação pósoperatória tardia, concluímos que a mucosetomia esofágica com conservação da túnica muscular demonstrando baixa repercussão clínica quando das complicações presentes e não relacionadas diretamente à técnica de ressecção e, sim, conseqüentes à reconstrução do trânsito gastrointestinal, nos estimula a porpor esse procedimento com maior freqüência para que a maior experiência o torne ainda mais seguro.

Desse modo esperamos oferecer nova alterantiva para aqueles que consideravam a ressecabilidade do esôfago como a melhor forma de terapêutica do megaesôfago avançado.

\begin{abstract}
Background: To perform a long term evaluation the technique of the esophageal mucosectomy in patients with advanced mega esophagus. Method: 50 patients with advanced mega esophagus submitted the esophageal mucosectomy with conservation of muscular layer in period of January 1991 to December of 1997 underwent a late evaluation between 6 to 15 years after the surgical procedure. The age varied between 30 and 69 years (mean age of 53.5 years), 32 (64\%) of them were males. The quality of deglution, the presence of regurgitation, alterations of the intestinal habit, the heavy evolution, the satisfaction with the surgery and with regard to normal work were assessed in all patients. Additionally, the morphological and functional evaluation with a contrast radiological study, upper digestive endoscopy and the thoracic CT scan were also performed. Each of the parameters were considered as good, excellent, regular and bad in accordance with total of the assigned points. Results: In global clinical evaluation, 44 patients (88\%) were considered as between excellent and good, while regular and bad in the others. In a global radiological evaluation, excellent and good had been presented in 47 patients (94\%) and regular and bad in the others. In global endoscopic evaluation, 45 patients (90\%) had excellent and good results, while regular and bad in the others. In relation the thoracic CT scan, 31 patients were considered good in all patients where the reconstruction of transit was carried through by the retroesternal route and excellent and good in all the patients where the reconstruction was through the transmediastinal route. Conclusion: The long term evaluation of patients submitted to esophageal mucosectomy for advanced mega esophagus showed excellent and good results in morphological, functional and clinical results in the majority of them.
\end{abstract}

Key words: Esophagus/surgery; Esofagectomy; Esophageal achalasia.

\section{REFERÊNCIAS}

1. Ferreira EAB. Esofagectomia subtotal, por via cérvico-abdominal combinada: sua possível utilização no megaesôfago. Rev Paul Med. 1973;82(3/4):133-4.

2. Ferreira EAB. Esofagogastroplastia e esofagocoloplastia transmediastinal posterior sem toracotomia. Rev Paul Med. 1974;84:142.

3. Ferreira EAB. Esofagectomia subtotal e esofagogastroplastia transmediastinal posterior sem toracotomia no tratamento do megaesôfago [Dissertação]. São Paulo (SP): Universidade de São Paulo; 1975.

4. Pinotti HW. Acesso extrapleural ao esôfago por frenolaparatomia. AMB Rev Assoc Med Bras. 1976;22(2):57-60.

5. Pinotti HW. Esofagectomia subtotal por túnel transmediastinal sem toracotomia. AMB Rev Assoc Med Bras. 1977;23(11):395-8.
6. Camara-Lopes LH. Câncer do esôfago como complicação de megaesôfago. Rev Paul Med. 1960;56(2):93.

7. Goodman P, Scott LD, Verani RR, Berggreen CC. Esophageal adenocarcinoma in a patient with surgically treated achalasia. Dig Dis Sci. 1990;35(12):1549-52.

8. Aquino JLB de, Moraes SP de, Martinez SE. Esofagectomia submucosa. Acta Cir Bras. 1989;4(1):64.

9. Aquino JLB. Tratamento do megaesôfago pela mucosectomia com conservação da túnica muscular esofágica por via cervicoabdominal [Dissertação]. Campinas (SP): Universidade Estadual de Campinas; 1996.

10. Aquino JLB, Reis Neto JA, Muraro CLPM, Camargo JGT. Mucosectomia esofágica no tratamento do megaesôfago avançado: análise de 60 casos. Rev Col Bras Cir. 2000;27(2):109-16.

11. Dantas RO, Godoy, RA de, Meneghelli UG. Segmental absence of contraction in the upper third of the esophagus. Arq Gastroenterol.1983;20(2):60-2. 
12. Kunzle JE, Ziliotto Júnior A. Estudo das anastomoses esofágicas cervicais após esofagectomia. Rev Col Bras Cir. 1985;12(2):425.

13. Chaib AS, Pinotti HW, Sakai P. Estômago intratorácico: estudo das alterações estruturais e funcionais e dos resultados a longo prazo. Rev Assoc Med Bras. 1980;26(1):33.

14. Ferraz EM, Bacelar TS, Ferreira Filho HA, et al. Tratamento cirúrgico do megaesôfago chagásico: avaliação de 60 casos. Ann Paul Med Cir. 1981;108(4):9-18.

15. Orringer MB, Stirling MC. Esophageal resection for achalasia: indications and results. Ann Thorac Surg. 1989;47(3):340-5.

16. Pinotti HW, Pollara WM, Raia AA. Tratamento cirúrgico do megaesôfago avançado pela esofagectomia subtotal por via cérvico-abdominal sem toracotomia com abertura do diafragma. Rev Assoc Med Bras. 1980;26(10):339-42.

17. Andreollo NA, Earlam RJ. Heller's myotomy for achalasia: is an added anti-reflux procedure necessary? Br J Surg. 1987;74(9):7659.

18. Miller H, Lam KH, Ong GB. Observations of pressure waves in stomach, jejunal, and colonic loops used to replace the esophagus. Surgery. 1975;78(5):543-51.

19. Wilbur BG, Kelly KA. Effect of proximal gastric, complete gastric, and truncal vagotomy on canine gastric electric activity, motility, and emptying. Ann Surg. 1973;178(3):295-303.
20. Rocha JRM. Tratamento do megaesôfago avançado pela esofagogastroplastia. Avaliação clínica e estudo da secreção do estômago e dos níveis séricos de pepsinogênio e gastrina [Dissertação]. São Paulo (SP): Universidade de São Paulo; 1986.

21. Rocha JM. Megaesôfago chagásico: continuamos a ressecar? In: Semana Brasileira de Aparelho Digestivo, 5, Recife, 2004. Anais.. p.40.

22. Fonseca A, Alves JCM, Lima MR et al. Esofagectomia sem toracotomia - Estudo de 10 casos. Rev Bras Cir. 1988;78(5):281-3.

Como citar este artigo:

Aquino JLB, Said MM, Fernandes PR. Avaliação tardia da mucosectomia esofágica com conservação da túnica muscular em pacientes com megaesôfago avançado. Rev Col Bras Cir. [periódico na Internet] 2007;34(1). Disponível em URL: http://www.scielo.br/rcbc

Endereço para correspondência:

José Luís Braga de Aquino

Rua Boaventura do Amaral, 1250/10 andar

Centro

13015-192 - Campinas - SP

E-mail:jlaquino@sigmanet.com.br 